\title{
Primary care management of asthma using written management plans and asthma clinics: evidence of effectiveness from recent Cochrane systematic reviews
}

\section{Felix S F Ram}

\begin{abstract}
Introduction
Over the last decade, much progress has been made in the management of asthma. Many countries and professional organisations have published asthma management guidelines that recognise the critical role of non-pharmacological components of asthma management in particular patient education, regular medical review and the provision of asthma management plans. ${ }^{1-4}$
\end{abstract}

However, despite substantial advances in our understanding of this disease and its management an unacceptably high level of morbidity and mortality related to asthma persists. There is a generally held belief that asthma management plans and asthma clinics providing education and regular review improves patient knowledge and disease outcomes. A number of controlled trials have been conducted to identify the effectiveness of these interventions however the results have been conflicting. This article summarises the findings from three recently completed Cochrane systematic reviews on the effectiveness of written asthma action plans, selfmanagement plans and primary care based asthma clinics. ${ }^{5-7}$ All Cochrane systematic reviews are conducted using the highest possible methodological quality, employing a transparent process and with duplication by independent persons of the process involved in the assembly of the review in order to minimise any potential for biases. $^{8}$

\section{Written asthma action plans}

A recent Cochrane review ${ }^{5}$ addressed the question of whether providing written management instructions to patients influences asthma outcome measurements. This review addressed the provision of written management plans only, and did not include studies that involved additional methods used for selfmanagement including asthma education or regular medical review, which was addressed in an earlier and recently updated Cochrane review. ${ }^{7}$ Both these reviews are discussed in this paper, since although each review addressed separate questions, they both dealt with issues relevant to the management of asthma in the primary care setting.

The new review of the effectiveness of individual written management plans examined the benefit of providing written management plans on the following outcomes: hospitalisation, emergency department visits, oral corticosteroid use, lung function, days lost from school or work, unscheduled doctor visits and respiratory tract infections. Six trials were identified as meeting the entry criteria for the review (Table 1). Some of the trials compared two different approaches (peak flow compared to symptom based plans), whilst others compared written plans with no written plans (Table 2). None of the reported outcomes in this review showed a statistically significant effect, whether written management plans were compared to no written plans or when peak flow based plans were compared to symptom based plans. There were too few data from the included trials to confirm whether or not written asthma management plans alone are effective in improving asthma outcomes.

\section{Self-management involving written plans, regular medical review and self-monitoring}

The objective of this review was to assess the effects of self-management plans (written asthma action plans, educational programmes, regular health practitioner review) on health outcomes in adults with asthma. ${ }^{7}$ Studies were included that compared selfmanagement plans to usual care. Interventions were categorised according to whether or not they involved a written action plan, regular medical review, selfmonitoring of peak expiratory flow or symptoms and/or asthma education. Written action plans involved an individualised written plan produced for the purpose of patient self-management of their asthma. Regular review was characterised by regular consultation with a doctor or nurse for the purpose of reviewing the patients' asthma status and medications. Self-monitoring was characterised by regular measurement of either peak expiratory flow or symptoms. Asthma education programme involved the transfer of information about asthma using either written, verbal, visual or audio means. A previous review dealt with trials of limited (information only) education interventions ${ }^{9}$ and concluded that education did not have a significant effect when administered without an action plan, self-monitoring or regular review. Education is considered to be necessary "to empower and help patients gain the motivation, skills and confidence in order to take control of their asthma". 4

The review included thirty-six trials that addressed a combination of methods for asthma self-management and regular review. ${ }^{7}$ The inclusion criteria for trial selection are listed in Table 1 and the characteristics of all trials included in the review are summarised in Table 2. The results are reported as relative risks (RR) with their $95 \%$ confidence intervals $(95 \% \mathrm{CI})$. Selfmanagement education reduced hospitalisations (RR $0.64,95 \% \mathrm{CI} 0.50$ to 0.82 ); emergency room visits ( $\mathrm{RR}$ $0.82,95 \% \mathrm{CI} 0.73$ to 0.94$)$; unscheduled visits to the doctor (RR $0.68,95 \% \mathrm{CI} 0.56$ to 0.81 ); days off work or school (RRR $0.79,95 \% \mathrm{CI} 0.67$ to 0.93 ) and nocturnal asthma (RR $0.67,95 \%$ CI 0.0 .56 to 0.79 ). Quality of life also improved significantly (standard mean difference $0.29,95 \% \mathrm{CI} 0.11$ to 0.47 ). However, measures of lung function did not change significantly. 
Self-management programmes that involved a written action plan showed much greater reduction in hospitalisation than those that did not (OR 0.35, $95 \%$ CI 0.18 to 0.68 ). People who managed their asthma by self-adjustment of their medications using an individualised written plan had better lung function than those whose medications were adjusted by a doctor. This review concluded that training in asthma self-management with a written action plan which involves self-monitoring by either peak expiratory flow or symptoms coupled with regular medical review, appears to improve health outcomes for adults

\section{Table 1: Trial inclusion criteria for each of the reviews.}

\section{Written individualised management plans for asthma in children and adults}

Types of trials: To be selected trials had to be randomised controlled trials. Patients in these trials must have been given individualised written instructions about the actions required for regular asthma management and/or the actions to take in the event of an asthma exacerbation. The only difference between the intervention and control groups was the provision of individualised written instructions.

Types of participants: Patients were adults and children who had a clinical diagnosis of asthma and had written instructions given to them by a doctor (or other health care professional) and requiring frequent use of asthma medications to manage their asthma.

Types of interventions: Trials were considered if the only intervention in the active group was the provision of written management plans. Different types of written management plans (peak flow versus symptom based) were also considered. Studies involving multiple interventions were excluded.
Self-management education and regular
practitioner review for adults with asthm

Types of trials: Trials were included if they were randomised controlled trials or controlled clinical trials which studied the effects of asthma education, regular medical review and self-monitoring on health outcomes in adults with asthma.

Types of participants: Adult patients were only considered who had a confirmed diagnosis of asthma according to objective criteria or internationally accepted guidelines.

Types of interventions: The interventions were categorised in the following manner according to whether or not they involved a written action plan, regular medical review, self-monitoring of peak expiratory flow or symptoms and/or asthma education or a mixture of all three.

\section{Primary care based clinics for asthma}

Types of trials: Only randomised controlled trials of any duration were considered for inclusion. Trials must have recruited patients into a regular primary care based asthma clinic run by either a nurse or other relevant health care professional.

Types of participants: Patients with asthma who must be participants in a primary care led, organised, and structured asthma clinic. Comparisons can be made between different types of care e.g. nonorganised or best clinical practice or alternative methods of primary care led structured care process.

Types of interventions: Primary care based practices, which offer a pro-active system of care by organised asthma clinics within the primary care setting. Practices that undertake shared care with hospital services were also considered for inclusion.

\section{Table 2: Summary characteristics of trials included in each of the reviews:}

\section{Written individualised action plans for asthma in children and adults}

Primary comparison: Individualised written asthma management plan versus control participants who received no written instructions.

Types of participants: All trials included patients with well-established asthma. Mean age for patients ranged from 28 to 36 years. Patients who previously had a written action plan or those requiring $>7.5 \mathrm{mg} /$ day of prednisone to control their asthma were excluded.

Setting: Trials had moderate sample sizes ranging from 72 to 150 patients. Three studies were conducted in tertiary hospitals three studies were conducted in primary care settings through family physicians.

Types of intervention: The types of intervention varied between studies. They included either peak flow or symptom based written plans compared to controls or both types of plans compared directly against each other.

Trial duration: The duration of intervention in three trials was 6 months and in another three, it was 12 months.

\section{Self-management education and regular practitioner review for adults with asthma}

Primary comparison: Self-management (involving written management plans, regular medical review and self monitoring) versus usual medical care with no self-management.

Types of participants: Adults with asthma defined by doctor's diagnosis or objective criteria or according to American Thoracic Society guidelines).
Primary care based clinics for asthma

Primary comparison: Primary care based asthma clinics, which offer a pro-active system of care compared to no asthma clinics.

Types of participants: Patients with asthma who are participants in a regular primary care based, organised, and structured asthma clinic.
Setting: 50 to 500 patients were included in each trial. Thirteen trials were conducted in primary care settings, nine in secondary care and four trials did not report trial setting.

Types of intervention: The interventions were categorised according to whether or not they involved a written action plan, regular medical review, self-monitoring of peak expiratory flow or symptoms and/or asthma education.

Trial duration: The duration of intervention varied greatly in the included trials as they varied from one hour to 12 months.
Setting: 195 adult patients with mean age of 26-27 yrs. Eight primary care practices (staffed by 42 general practitioners) in South Australia.

Types of intervention: Each primary care practice operated one three-hour asthma clinic session per week. The asthma educators in the trial were registered nurses with extensive experience in respiratory care.

Trial duration: There was only one included trial in this review, which had duration of 6 months. 
with asthma. Training programmes which enable people to adjust their medication using a written action plan appear to be more effective than other forms of asthma self-management. However, as discussed in the previous review ${ }^{5}$ written action plans alone have not been shown to significantly improve asthma outcomes. Therefore, evidence to date suggests that written action plans should be used in combination with asthma education and regular medical review if they are to be effective.

\section{Primary care based asthma clinics}

In addition to the provision of asthma management plans there has been a proliferation of primary care based asthma clinics in the hope that they would also improve asthma outcomes. The contract for general practitioners, introduced to the UK National Health Service in 1990, provided remuneration for chronic disease clinics, resulting in pro-active care being offered to practice populations of asthma patients. However, the effectiveness of this system has received only limited evaluation by today's standards of rigorous evidence-based medicine. Although Health Authorities in the UK generally require audit data on asthma clinics, the focus is usually on structure and process rather than outcomes. In 1993, the National Asthma Task Force set out to establish a baseline of work done in community asthma clinics and called for further evaluation of the effectiveness of asthma clinics. ${ }^{10}$ However, since most practices now implement nurse-run asthma clinics, it has been argued that it may be impossible to undertake a randomised controlled trial. ${ }^{10}$

Unfortunately, little is known about the effectiveness of primary care based asthma clinics and their potential to improve asthma outcomes. Even less is known about patients' views on these clinics. A primary care based asthma clinic is normally led by a nurse, and usually has support from the patient's general practitioner and involves organised recall of patients with asthma for symptom review and input from a variety of educational models. The effectiveness of this system has received only limited evaluation in the form of randomised controlled trials compared to usual care. A recent Cochrane review addressed the effectiveness of organised care through primary care based asthma clinics. ${ }^{6}$ Unfortunately, only one trial met the review's inclusion criteria (Tables 1 and 2). This trial provided 11 outcome measures of which two showed a significant effect of the intervention. More patients in the intervention group had peak flow meters (RR $1.30,95 \%$ CI 1.05 to 1.61) and fewer patients in the intervention group were likely to wake up at night as a result of their asthma (RR $0.30,95 \% \mathrm{CI} 0.16$ to 0.81 ). Due to limited evidence of benefit from primary care based asthma clinics firm conclusions cannot be formed until further good quality trials have been conducted.

\section{Applicability of findings}

Although, some of the included trials in the reviews mentioned above were conducted in a secondary care setting, the majority of included trials were conducted in primary care. In the self-management review ${ }^{7}$ the majority of the trials took place in the secondary care setting in people with moderate to severe asthma. Few trials have taken place in primary care, the majority of which have been negative. More work is needed to convincingly demonstrate that the findings of this review are applicable to the general population of people with asthma in primary care. In the written management plan review ${ }^{5}$ three trials each were conducted in secondary and primary care. In the third review on primary care based asthma clinics ${ }^{6}$ there was only one included trial, which was conducted in primary care (Table 2). However, we have no reason to believe that the results of the included trials conducted in secondary care would not also apply to patients in the primary care setting. Included trials which were conducted in secondary care utilised the facilities and staff of the secondary care institute however in most instances patients were recruited from the community and those patients who were being discharged into the community after a period as inpatient. Combining these different trial results in a meta-analysis did not produce statistical heterogeneity, adding further to the argument that the results from the two settings are not mutually exclusive.

\section{Conclusion}

Although the provision of personalised written asthma actions plans to patients is good clinical practice there is currently not enough evidence from randomised trials to show that written asthma action plans produce better outcomes than no written plan. There is a need to conduct further trials that specifically address the effectiveness of written asthma action plans.

Asthma self management plans are of significant benefit to patients. Patients who are educated about their asthma, visited the doctor or nurse regularly and who used a written action plan had fewer visits to the emergency room, less hospital admissions, less unschedule docotor visits, less days of work or school and less nocturnal asthma. Training programmes that enable people to adjust their medication using a written action plan appear to be more effective than other forms of asthma self-management.

Traditionally, the educational role of asthma clinics has been accorded great importance in literature and is emphasised in training courses attended by the majority of asthma nurses. Despite the proliferation of primary care based asthma clinics throughout the UK, there is very little evidence from randomised trials on their effectiveness in improving asthma outcomes. Further research is needed in order to improve our understanding of the effectiveness of primary care based asthma clinics.

The questions remain then: how best to manage patients with asthma in primary care; in organised asthma clinics?, in normal surgery time with planned follow-up?, or opportunistically on an acute basis? Accessing and using evidence in this area is likely to have important resource implications. 


\section{Review}

For personal use only.

Not to be reproduced without the permission of the Primary Care Respiratory Journal

\section{Felix S F Ram}

Research Fellow in

Respiratory Medicine

Correspondence to:

St. George's Hospital

Medical School

Department of

Physiological Medicine

University of London

Level 0, Jenner Wing

Cranmer Terrace, Tooting

London, SW17 0RE

Email: fram@sghms.ac.uk

Date submitted: $31 / 10 / 02$

Date Accepted: 10/02/03

Prim Care Resp J 2003;

12(1):21-24

\section{Acknowledgements:}

Dr Felix S F Ram is supported by a fellowship from the Netherlands Asthma Foundation, The Netherlands. References

1. BTS/SIGN. British guidelines on the management of asthma. Thorax 2003;58(Suppl 1):1-94.

2. NAC. Asthma Management Handbook. Melbourne, Australia: National Asthma Campaign, 1996. 3. NIH. Expert panel report II: Guidelines for the diagnosis and management of asthma. Bethesda, USA: National Institutes of Health. National Asthma Education and Prevention Program, 1997.

4. Annonymous. Asthma Management Handbook.

Melbourne: National Asthma Campaign, Australia, 1996.

5. Toelle B, Ram FSF. Written individualised management plans for asthma (Cochrane Review). Issue 4 ed: The Cochrane Library, Update Software, 2002.
6. Fay JK, Jones A, Ram FSF. Primary care based clinics for asthma (Cochrane Review). Issue 4: The Cochrane Library, Update Software, 2002.

7. Gibson PG, Coughlan J, Wilson AJ, Abramson M, Bauman A, Hensley MJ, et al. Self-management education and regular practitioner review for adults with asthma (Cochrane Review). Issue 4 ed: The

Cochrane Library, Update Software, 2002.

8. Ram FSF. The art of producing quality evidencebased systematic reviews. Prim Care Resp J 2002;11(4):117-9.

9. Gibson PG, Coughlan J, Wilson AJ, Hensley MJ, Abramson M, Bauman A, et al. The effects of limited (information-only) asthma education on health outcomes of adults with asthma (Cochrane Review). Issue 1: The Cochrane Library, Update Software, 2002.

10. Jones KP, Mullee MA. Proactive, nurse-run asthma care in general practice reduces asthma morbidity: scientific fact or medical assumption? Brit J Gen Prac 1995;45:497-9

\section{Calls for nomination for committee membership}

A vacancy will become available on the GPIAG committee from June 2003. If you are interested in becoming a committee member simply contact the GPIAG offices for an application form, complete the details and obtain endorsement from a fellow member.

Election to the committee will be by majority vote of the membership.

Only GPIAG full members are entitled to stand for committee membership. The term of office is three years and you will be expected to attend all committee meetings and play an active role in the ongoing growth and development of the group and its activities.

For further information or to obtain an application form contact the GPIAG secretariat:

Edgbaston House, 3 Duchess Place, Edgbaston, Birmingham, B16 8NH

$$
\begin{gathered}
\text { Telephone +44 (0)1214548219 Facsimile: +44 (0)121 } 4541190 \\
\text { email: info@gpiag.org }
\end{gathered}
$$

